\title{
NRG1: a cinderella fusion in lung cancer?
}

\author{
Lucia Anna Muscarella ${ }^{1}$ \& Antonio Rossi*,2 \\ ${ }^{1}$ Laboratory of Oncology, Scientific Institute for Research \& Health Care (IRCCS) 'Casa Sollievo della Sofferenza', San Giovanni \\ Rotondo (FG), Italy \\ ${ }^{2}$ Division of Medical Oncology, Scientific Institute for Research \& Health Care (IRCCS), Casa Sollievo della Sofferenza Hospital, Viale \\ Cappuccini 1, 71013, San Giovanni Rotondo (FG), Italy \\ *Author for correspondence: Tel.: +39 0882410 716; Fax: +39 0882204 095; arossi_it@yahoo.it
}
"Since NRG1 fusions act through the activation of the ERBB receptor, blocking the activity of the NRG1-ERBB-PI3K-AKT pathway might be the best strategy for the treatment of NRG1-fused tumors."

First draft submitted: 11 November 2017; Accepted for publication: 21 November 2017; Published online: 5 January 2018

Keywords: ErbB • NRG1 fusion • NSCLC

The amazing bridge between gene fusions and lung cancer has been greatly consolidated during the last decades. The identification of $A L K$ and $R O S 1$ rearrangements gives the great opportunity to rewrite the standard-of-care for a portion of advanced non-small-cell lung cancer (NSCLC) patients. Despite their very low incidence, these well-known genomic rearrangements share common features strictly associated to specific phenotypes of lung adenocarcinoma. This makes them ideal for diagnostic procedures, patients' stratification and therapies $[1,2]$. In this scenario, the finding of further intriguing targetable gene fusions, despite with a low incidence (1-2\%) in NSCLC, is now increasing and it is watched with attention by the clinical community [3].

Recently, the NRG1 gene has been described as a new molecular feature of NSCLC [4]. The NRG1 gene is located at the long arm of chromosome 10 (10q23.1 region) and encodes for the neuregulin 1, a growth factor belonging to the complex family of proteins also called heregulins. These proteins are structurally related to the stimulation of ERBB receptors tyrosine kinase activity and EGF signals. Specifically, the NRG1-receptor binding induces the phosphorylation of the intrinsic kinase domains of ERBB3 and stimulates its dimerization with ERBB2 receptor and the activation of the downstream PI3K-AKT and MAPK pathways [5].

The neuronal isoform NRG1 III- $\beta 3$ is generally not expressed in normal lung tissue, but it was found to be ectopically activated in lung tumor cells by $N R G 1$ genomic rearrangements involving mainly CD74 and SLC3A2 genes [6]. It is the first fusion associated with the mucinous subtype of lung adenocarcinoma with an occurrence ranging from 8 to $27 \%$. By contrast, NRG1-gene fusions occur in 1-2\% of NSCLC and occasionally reported in other solid tumors [7]. It has been more extensively investigated in Asian than Caucasian lung cancer patients in whom it has been not yet fully elucidated and remains only partially understood [6].

ERBB signaling is one of the most deregulated lung cancer cascades. ERBB3 is not frequently affected by mutations or amplifications in lung cancer. Furthermore, MET or HER2 amplifications, which represent additional mechanisms of ERBB3 activation in lung tumors, rarely occur. Thus, the overproduction of NRG1 ligands could represent one of the leading mechanisms by which lung cancer cells aberrantly activate ERBB3-related receptor tyrosine kinase signaling.

The first suggestion of a real clinical utility of NRG1 fusion comes by analyzing a large sample of mucinous lung adenocarcinoma in Asians for the NRG1 breaks. In fact, patients with stage I disease harboring tumors with $N R G 1$ fusions showed an inferior overall survival and a trend toward a shorter disease-free survival compared with those without $N R G 1$ fusions [8]. The powerfulness of this fusion has been highlighted in vitro. In fact, the expression of $C D 74-N R G 1$ fusion gene is able to promote cancer stem cell properties and it is involved in stem cell function of several types of cancers, including lung cancer. These data imply the existence of a mechanism by which the activated ERBB receptors contribute to the acquisition of cancer stem cell-like characteristics together with the ability of cancer cells to develop a resistance to chemotherapy [9]. Moreover, in the absence of RAS pathway 
mutations, NRG1 overexpression can play a major role in the primary cetuximab resistance in colon cancer cells and in primary resistance to trastuzumab in HER2 overexpressing breast cancer cells [10,11].

Of interest, NRG1 fusions have been proved to be coexistent with $A L K$ fusion or RAS mutation in NSCLC patients, both in primary and in metastatic sites of lung tumors [12-14]. Moreover, it has been recently considered as a potential mechanism of resistance after treatment with tyrosine kinase inhibitors in $A L K$-rearranged NSCLC cell lines. In fact, by using primary cultures of cancer cells from pleural effusion of an $A L K$-positive lung cancer patient, the increase of the NRG1 ligand levels and the consequent activation of ERBB3 pathway has been directly related to resistance to crizotinib treatment [15]. This assumption has been further confirmed showing that, under treatment with second-generation ALK inhibitors, NSCLC cells activated the EGFR family pathways directly through the NRG1-ERBB3-EGFR activation axis [16]. In support to these in vitro reports, the onset of the SLC3A2-NRG1 gene fusion during the natural history of two invasive mucinous lung adenocarcinoma in Asiatic patients has been described. These heavily pretreated patients received the combination of lumretuzumab, a monoclonal anti-ERBB3 antibody, plus erlotinib, an anti-EGFR small molecule, showing tumor shrinkage [17]. Furthermore, the use of afatinib, a pan-ERBB-family kinase inhibitor, in NRG1-positive samples resulted in a surprisingly durable response in patients with lung adenocarcinoma and cholangiocarcinoma $[7,18]$.

ERBB3 overexpression actually represents one of the targets of greater interest for the current pharmacological studies. In fact, its activation through phosphorylation has been detected in various cancers including metastatic lung carcinoma in presence of acquired resistance to other ERBB family inhibitors [19]. Moreover, due to its inactive tyrosine kinase domain, ERBB3 has been reported to have a critical role in the dimerization process in the context of acquired de novo resistance to ERBB3 targeted therapies. However, despite the well-tested efficacy of EGFR and HER2 inhibitors, ERBB3 specific upregulation has not been yet targeted with clear clinical efficacy. Of all the anti-ERBB3 agents, patritumab is in advanced clinical development, being currently investigated in a Phase III trial for treatment of NSCLC (JapicCTI-101262) [20]. Additional trials are investigating neratinib, alone and in combination with temsirolimus (NCT01827267) and trastuzumab emtansine (T-DM1; NCT02289833), in HER2 molecular profiled advanced NSCLC. Of great interest is MM-121. An ongoing open-label trial is investigating MM-121, a fully human monoclonal antibody targeting specifically ERBB3, in combination with docetaxel or pemetrexed compared with docetaxel or pemetrexed alone, in patients with heregulin-positive, advanced NSCLC with primary end point overall survival (NCT02387216).

Overall, NRG1 fusions could represent new potential molecular alterations able to predict the therapy activity in a specific lung adenocarcinoma subtype. Since $N R G 1$ fusions act through the activation of the ERBB receptor, blocking the activity of the NRG1-ERBB-PI3K-AKT pathway might be the best strategy for the treatment of $N R G 1$-fused tumors.

\section{Financial \& competing interests disclosure}

This work was supported by the Italian Ministry of Health (Ricerca Corrente, RC1703LO41 to LA Muscarella and RC1703ON39 to A Rossi), by the ' $5 \times 1000$ ' voluntary contributions and by the AIRC/MFAG grant 12983 (to LA Muscarella) and FBNC Grant 2015-2016 to LA Muscarella. The authors have no other relevant affiliations or financial involvement with any organization or entity with a financial interest in or financial conflict with the subject matter or materials discussed in the manuscript apart from those disclosed.

No writing assistance was utilized in the production of this manuscript.

\section{References}

1 Kwak El, Bang YJ, Camidge DR et al. Anaplastic lymphoma kinase inhibition in non-small-cell lung cancer. N. Engl. J. Med. 363(18), 1693-1703 (2010).

2 Davies KD, Le AT, Theodoro MF et al. Identifying and targeting ROS1 gene fusions in non-small-cell lung cancer. Clin. Cancer Res. 18(17), 4570-4579 (2012).

3 Schram AM, Chang MT, Jonhsson P, Drilon A. Fusions in solid tumours: diagnostic strategies, targeted therapy and acquired resistance. Nat. Rev. Clin. Oncol.doi: 10.1038/nrclinonc.2017.127 (2017) (Epub ahead of print).

4 Fernandez-Cuesta L, Plenker D, Osada H et al. CD74-NRG1 fusions in lung adenocarcinoma. Cancer Discov. 4(4), 415-422 (2014).

5 Yarden Y, Pines G. The ERBB network: at last, cancer therapy meets systems biology. Nat. Rev. Cancer 12(8), 553-563 (2012).

6 Trombetta D, Rossi A, Fabrizio FP et al. NRG1-ErbB lost in translation: a new paradigm for lung cancer? Curr. Med. Chem.doi: 10.2174/0929867324666170911170554 (2017) (Epub ahead of print). 
7 Jones MR, Lim H, Shen Y et al. Successful targeting of the NRG1 pathway indicates novel treatment strategy for metastatic cancer. Ann. Oncol. doi: 10.1093/annonc/mdx523 (2017) (Epub ahead of print).

8 Shin DH, Lee D, Hong DW et al. Oncogenic function and clinical implications of SLC3A2-NRG1 fusion in invasive mucinous adenocarcinoma of the lung. Oncotarget 7(43), 69450-69465 (2016).

9 Murayama T, Nakaoku T, Enari $\mathrm{M}$ et al. Oncogenic fusion gene CD74-NRG1 confers cancer stem cell-like properties in lung cancer through a IGF2 autocrine/paracrine circuit. Cancer Res. 76(4), 974-983 (2016).

10 Luraghi P, Bigatto V, Cipriano E et al. A molecularly annotated model of patient-derived colon cancer stem-like cells to assess genetic and non-genetic mechanisms of resistance to anti-EGFR therapy. Clin. Cancer. Res. doi: 10.1158/1078-0432.CCR-17-2151 (2017) (Epub ahead of print).

11 Yang L, Li Y, Shen E et al. NRG1-dependent activation of HER3 induces primary resistance to trastuzumab in HER2-overexpressing breast cancer cells. Int. J. Oncol. 51(5), 1553-1562 (2017).

12 Xia D, Le LP, Iafrate AJ, Lennerz J. KIF13B-NRG1 gene fusion and KRAS amplification in a case of natural progression of lung cancer. Int. J. Surg. Pathol. 25(3), 238-240 (2017).

13 Muscarella LA, Trombetta D, Fabrizio FP et al. ALK and NRG1 fusions coexist in a patient with signet ring cell lung adenocarcinoma. J. Thorac. Oncol. 12(10), E161-E163 (2017).

14 Dhanasekaran SM, Balbin OA, Chen G et al. Transcriptome meta-analysis of lung cancer reveals recurrent aberrations in NRG1 and Hippo pathway genes. Nat. Commun. 5, 5893 (2014).

15 Dong X, Fernandez-Salas E, Li E, Wang S. Elucidation of resistance mechanisms to second-generation ALK inhibitors alectinib and ceritinib in non-small-cell lung cancer cells. Neoplasia 18(3), 162-171 (2016).

16 Kimura M, Endo H, Inoue T et al. Analysis of ERBB ligand-induced resistance mechanism to crizotinib by primary culture of lung adenocarcinoma with EML4-ALK fusion gene. J. Thorac. Oncol. 10(3), 527-530 (2015).

17 Ji-Youn H, Kun Young L, Jin Young K et al. EGFR and HER3 inhibition- A novel therapy for invasive mucinous non-small-cell lung cancer harbouring an NRG1 fusion gene. J. Thorac. Oncol. 12(S1), S669, Abstract P3.02C-006 (2016).

18 Gay ND, Wang Y, Beadling C et al. Durable response to afatinib in lung adenocarcinoma harboring NRG1 gene fusions. J. Thorac. Oncol. 12(8), E107-E110 (2017).

19 Sun M, Behrens C, Feng L et al. HER family receptor abnormalities in lung cancer brain metastases and corresponding primary tumors. Clin. Cancer Res. 15(15), 4829-4837 (2009).

20 Malm M, Frejd FY, Ståhl S, Löfblom J. Targeting HER3 using mono- and bispecific antibodies or alternative scaffolds. MAbs 8(7), 1195-1209 (2016). 